\title{
Utilização do pó de fumo no substrato para produção de mudas de tucaneira
}

\author{
Utilization of the powder smoke in the substrate for production \\ of tucaneira seedlings
}

\section{Tatiele Anete Bergamo Fenilli ${ }^{[a]}$, Lauri Amândio Schorn ${ }^{[\mathrm{b}]}$, Susan Karla Nasato ${ }^{[\mathrm{c}]}$}

[a] Engenheira agrônoma, professora Doutora da Universidade Regional de Blumenau (FURB), Departamento de Engenharia Florestal, Blumenau, SC - Brasil, e-mail: tfenilli@furb.br

[b] Engenheiro florestal, professor Doutor da Universidade Regional de Blumenau (FURB), Departamento de Engenharia Florestal, Blumenau, SC - Brasil, e-mail: lschorn@furb.br

[c] Bolsista de Iniciação Científica da Universidade Regional de Blumenau (FURB), Departamento de Engenharia Florestal, Blumenau, SC - Brasil, e-mail: susan_florestal@yahoo.com.br

\section{Resumo}

O objetivo do estudo foi avaliar o crescimento de mudas da espécie Citharexylum myrianthum utilizando, na formulação do substrato, resíduo de pó de fumo. A pesquisa foi instalada no Horto Florestal do Departamento de Engenharia Florestal da Universidade Regional de Blumenau (FURB). As mudas foram produzidas em recipientes plásticos com volume de $1.462,5 \mathrm{~cm}^{3}$ de substrato. O delineamento experimental utilizado foi inteiramente casualizado com 5 tratamentos (testemunha, 10\%, 20\%, 30\% e $40 \%$ de pó de fumo no substrato) e 25 repetições de uma planta por parcela, totalizando 125 mudas. Após 40 dias da semeadura, foram avaliados os parâmetros: diâmetro do colo, altura total da parte aérea, comprimento de raiz, produção de biomassa do sistema radicial, da parte aérea e total, e as relações entre altura da parte aérea e produção de biomassa da parte aérea, biomassa da parte aérea e biomassa de raiz, e altura da parte aérea com diâmetro do colo, além do índice de qualidade de Dickson. Com os resultados, observou-se que as mudas de tucaneira responderam de maneira distinta às diferentes proporções de pó de fumo na formulação do substrato, e que as porcentagens maiores (30\% e 40\%) de pó de fumo na formulação afetaram seu crescimento e desenvolvimento.

Palavras-chave: Nutrição de plantas. Desenvolvimento. Produção de biomassa. Diâmetro do colo. Altura da planta. 


\begin{abstract}
The main goal of this project was to evaluate Citharexylum myrianthum seedlings growth using powder smoke residue in the substrate. The research was installed at the Horto of the Forest Engineering Department/FURB. The seedlings were produced in plastic containers with $1.462,5 \mathrm{~cm}^{3}$ of substrate. The experimental design was complete randomized with 5 treatments (control, 10, 20, 30 and $40 \%$ of powder smoked in the substrate), and 25 replications of one plant for plot, consisting of 125 seedlings. After 40 days sowing, some parameters were evaluated: base diameter, total height, root length, and root and shoot biomass production, and relations between total height and collar diameter, shoot biomass and root biomass and, total height with collar diameter, besides the quality index of Dickson. Results showed that Citharexylum myrianthum seedlings responded in distinctive way for the different proportions of powder smoke in the substrate, and the larger percentages (30\% and $40 \%$ ) of powder smoke residue affected their growth and development.
\end{abstract}

Keywords: Nutrition of plants. Development. Biomass production. Base diameter. Plant height.

\title{
Introdução
}

O Brasil possui grande diversidade vegetal, mas a falta de conscientização ecológica e a exploração de nossos recursos florestais têm acarretado prejuízos irreparáveis e espécies de grande valor ecológico estão em vias de extinção (LORENZI, 1998).

As necessidades e justificativas para o reflorestamento são muitas, podendo ser realizado com árvores de uso múltiplo em monocultivo ou em sistemas agroflorestais, o que varia de acordo com as características ambientais e socioeconômicas de cada região (FRANKE et al., 2000). Franke et al. (2000) e Porfírio da Silva e Mazuchowski (1999) indicam que a presença de árvores favorece os sistemas de produção agroflorestais em aspectos como a manutenção da ciclagem de nutrientes e o aumento na diversidade de espécies.

A tucaneira (Citharexylum myrianthum) é uma árvore nativa pioneira e rústica, muito comum na Floresta Atlântica, e é uma das primeiras a aparecer em áreas degradadas. Após o plantio em reflorestamento, um dos fatores na determinação da sobrevivência de uma espécie é seu desenvolvimento inicial (CARNEIRO, 1995; FONSECA, 2000).

A formação de mudas florestais de boa qualidade envolve os processos de germinação de sementes, iniciação radicular, formação do sistema radicular e parte aérea, os quais estão diretamente relacionados com características que definem o nível de eficiência dos substratos, tais como: aeração, drenagem, retenção de água e disponibilidade balanceada de nutrientes (GONÇALVES; POGGIANI, 1996; TRIGUEIRO; GERRINI, 2003).

Os substratos orgânicos utilizados na fase de viveiro em silvicultura são, na sua maioria, pobres em nutrientes essenciais ao crescimento da planta, e, nesse sentido, a fertilização é um dos fatores mais importantes para garantir o bom desenvolvimento das mudas. A utilização de um substrato que promova rápido crescimento inicial das mudas é fundamental para melhorar a tecnologia de produção na fase de viveiro, com uma expectativa de atender à demanda de mudas para um mercado em franca expansão (MORAIS et al., 1996).

Segundo Gonçalves et al. (2000), substratos adequados para a propagação de mudas com sementes e estacas podem ser obtidos a partir da mistura de 70\% a 80\% de um componente orgânico (esterco de bovino, casca de eucalipto ou pinus, bagaço de cana, lixo urbano, outros resíduos e húmus de minhoca), com $20 \%$ a $30 \%$ de um componente usado para elevar a macroporosidade (casca de arroz carbonizada, cinza de caldeira de biomassa, bagaço de cana carbonizado).

A produção e estocagem dos resíduos industriais nas empresas têm atingido valores elevados e causam preocupação em relação à preservação do meio ambiente. O destino do pó de fumo tem sido a lavoura dos fumicultores. Nesse sentido, o resíduo de pó de fumo serve como uma alternativa para a melhoria da fertilidade dos solos, podendo resultar em ganhos significativos de produtividade, devendo-se buscar soluções 
economicamente viáveis para minimizar os impactos causados pela sua adição sem tratamento adequado (CALDEIRA et al., 2007).

Atualmente, há grande necessidade em desenvolver estudos na utilização de resíduos industriais visando ao seu aproveitamento. No entanto, poucos são os estudos conduzidos no Brasil, principalmente com resíduo de pó de fumo como insumo na produção de mudas.

Nesse sentido, o objetivo deste trabalho foi avaliar a utilização do resíduo do pó de fumo na formulação de substrato para o desenvolvimento de mudas de tucaneira (Citharexylum myrianthum).

\section{Materiais e métodos}

A presente pesquisa foi instalada no viveiro do Horto Florestal do Departamento de Engenharia Florestal da Universidade Regional de Blumenau (FURB), no município de Gaspar, SC. A região possui clima Cwa, segundo a classificação do clima de Köppen, caracterizando clima subtropical úmido, com temperatura média de $19^{\circ} \mathrm{C}$ e pluviosidade média de $2000 \mathrm{~mm}$ /ano. O relevo é composto por planícies situadas próximas ao Rio Itajaí. As coordenadas geográficas de Gaspar são: latitude sul 2654'40.2" e longitude 4856'13.9".

Com base nas recomendações de mistura de componentes para composição de substratos sugeridas por Gonçalves et al. (2000), optou-se por trabalhar com proporções dos componentes do pó de fumo no substrato padrão utilizado no Horto Florestal.

O resíduo da industrialização do tabaco, pó de fumo, utilizado para a produção das mudas, foi adquirido da Souza Cruz S.A. de Blumenau, SC, e apresenta a seguinte composição química: textura $=28 \%$ de argila; $\mathrm{pH}=8,20 ;$ matéria orgânica $=10 \%$; fósforo total $=50 \mathrm{ppm} \mathrm{em} \mathrm{P}_{2} \mathrm{O}_{5}$; potássio solúvel em água $=995 \mathrm{ppm} \mathrm{em} \mathrm{K}_{2} \mathrm{O} ;$ cálcio $=7,40 \mathrm{cmol}_{\mathrm{c}} / 1$, magnésio $=7 \mathrm{cmol}_{\mathrm{c}} / \mathrm{l} ;$ sódio $=54 \mathrm{ppm} ; \mathrm{Al}=0 \mathrm{cmol}_{\mathrm{c}} / \mathrm{l}$; $\mathrm{H}+\mathrm{Al}=0,55 \mathrm{cmol}_{\mathrm{c}} / 1$; Soma Bases $=17,19 \mathrm{cmol}_{\mathrm{c}} / 1 ; \mathrm{CTC}=17,74 \mathrm{cmol}_{\mathrm{c}} / 1$ e saturação por bases $=96,90 \%$.

Foram utilizadas sementes coletadas de uma árvore da espécie Citharexylum myrianthum no município de Gaspar. As sementes foram extraídas após maceração dos frutos para seu despolpamento e secas à sombra. Como a espécie não apresenta dormência, não foi necessário o tratamento das sementes. O substrato padrão e o adubo (NPK) formulação 4-14-8 foram fornecidos pelo Horto Florestal.

As mudas foram produzidas em recipientes de plástico com altura $22 \mathrm{~cm}$ e diâmetro $9,2 \mathrm{~cm}(1.462,5$ $\mathrm{cm}^{3}$ de substrato). Os sacos plásticos foram preenchidos com técnica empregada pelo viveiro florestal da FURB, que consistiu em encher os recipientes com o substrato já misturado ao adubo contendo NPK (2 $\mathrm{kg}$ de 4-14-8 por $\mathrm{m}^{3}$ de mistura), realizando leve compactação manualmente, de forma que o substrato se acomodasse dentro dos recipientes e, em seguida, completando-se o volume destes. Foram semeadas diretamente duas sementes por recipiente em 6 de outubro de 2008. Após a germinação foi efetuado o desbaste, deixando-se uma muda por recipiente.

O delineamento experimental utilizado foi inteiramente casualizado com 5 tratamentos e 25 repetições de duas plantas por parcela, com apenas uma planta útil para análise estatística. Os tratamentos foram compostos por diferentes proporções do pó de fumo (Tabela 1). As análises químicas dos substratos utilizados no experimento se encontram na Tabela 2.

Após 40 dias da semeadura, em 14 de novembro de 2008 foram avaliadas as seguintes variáveis e suas relações: diâmetro do colo (D), altura da parte aérea (h), comprimento de raiz (CR), massa seca da parte aérea (MSPA), massa seca de raiz (MSR), relação entre a altura da parte aérea e o diâmetro do colo (h/D), relação entre a altura da parte aérea e a massa seca da parte aérea (h/MSPA) e relação entre a massa seca da parte aérea e a massa seca de raiz (MSPA/MSR) (GOMES et al., 2002). O diâmetro do colo foi medido ao nível do substrato com paquímetro digital. A altura da parte aérea e o comprimento de raiz após lavagem com água destilada e secagem em papel toalha ao ar livre sobre sombra foram mensurados com auxílio de uma régua graduada em $\mathrm{cm}$. A altura da parte aérea foi determinada a partir do nível do substrato até a ponta da última folha, e o comprimento de raiz foi medido a partir do nível do substrato até a ponta da raiz mais longa. Para a determinação da massa seca da parte aérea (folhas + caule) e da raiz em balança de precisão, as amostras foram colocadas em sacos de papel, e secas em estufa com temperatura média de $60^{\circ} \mathrm{C}$ até massa constante. 
A soma da MSPA com MSR forneceu a massa seca total (MST). Esses parâmetros foram utilizados para calcular o índice de qualidade de Dickson (IQD), de acordo com a seguinte fórmula (DICKSON et al., 1960):

$$
\mathrm{IQD}=\frac{\operatorname{MTS}(\mathrm{g})}{\mathrm{h}(\mathrm{cm}) \div \mathrm{D}(\mathrm{mm})+\operatorname{MSPA}(\mathrm{g}) \div \operatorname{MSR}(\mathrm{g})}
$$

Os dados analisados foram submetidos à análise de variância e as médias de cada tratamento foram comparadas pelo teste de Tukey a 5\% de probabilidade.

Tabela 1 - Tratamentos com suas respectivas formulações de substrato

\begin{tabular}{ll}
\hline Tratamento & Formulações \\
\hline $\mathbf{T}_{1 \text { (testemunha) }}$ & $\begin{array}{l}100 \% \text { substrato padrão }(50 \% \text { de terra de subsolo }+30 \% \text { casca de arroz }+ \\
+10 \% \text { composto de aves }+10 \% \text { composto bovino })+\mathrm{NPK}\end{array}$ \\
$\mathbf{T}_{2}$ & $90 \%$ substrato padrão $+10 \%$ resíduo de pó de fumo \\
$\mathbf{T}_{3}$ & $80 \%$ substrato padrão $+20 \%$ resíduo de pó de fumo \\
$\mathbf{T}_{4}$ & $70 \%$ substrato padrão $+30 \%$ resíduo de pó de fumo \\
$\mathbf{T}_{5}$ & $60 \%$ substrato padrão $+40 \%$ resíduo de pó de fumo \\
\hline
\end{tabular}

Tabela 2 - Análise química dos substratos utilizados na produção das mudas de Citharexylum myrianthum

\begin{tabular}{|c|c|c|c|c|c|c|}
\hline Determinação & $\mathbf{T}_{1}$ & $\mathbf{T}_{2}$ & $\mathbf{T}_{2}$ & $\mathbf{T}_{4}$ & $\mathbf{T}_{5}$ & Unidade \\
\hline Textura & 17,00 & 16,00 & 17,00 & 14,00 & 15,00 & $\%$ Argila \\
\hline $\mathrm{pH}$ & 4,20 & 5,70 & 6,10 & 6,60 & 6,90 & \\
\hline Índice SMP & 5,80 & 6,80 & 6,80 & 7,10 & 7,20 & \\
\hline Fósforo & $>50,00$ & $>50,00$ & $>50,00$ & $>50,00$ & $>50,00$ & ppm \\
\hline Potássio & 512,00 & 720,00 & 1140,00 & 980,00 & 1220,00 & ppm \\
\hline Mat. orgânica & 2,90 & 4,40 & 6,20 & 6,50 & 9,00 & $\%(\mathrm{~m} / \mathrm{v})$ \\
\hline Alumínio & 0,40 & 0,00 & 0,00 & 0,00 & 0,00 & $\mathrm{cmolc} / \mathrm{l}$ \\
\hline Cálcio & 4,30 & 6,60 & 6,80 & 7,90 & 8,80 & cmolc/1 \\
\hline Magnésio & 2,50 & 3,90 & 4,10 & 4,80 & 5,40 & cmolc/l \\
\hline Sódio & 40,00 & 48,00 & 48,00 & 54,00 & 56,00 & ppm \\
\hline $\mathrm{H}+\mathrm{Al}$ & 5,49 & 1,74 & 1,74 & 1,23 & 1,10 & cmolc/1 \\
\hline Soma bases-S & 8,29 & 12,56 & 14,04 & 14,45 & 17,58 & $\mathrm{cmolc} / \mathrm{l}$ \\
\hline СTC & 13,78 & 14,30 & 15,78 & 16,68 & 18,68 & cmolc/1 \\
\hline V\% & 60,16 & 87,83 & 88,97 & 92,63 & 94,11 & $\%$ \\
\hline
\end{tabular}

Rev. Acad., Ciênc. Agrár. Ambient., Curitiba, v. 8, n. 2, p. 183-190, abr./jun. 2010 


\section{Resultados e discussão}

A Tabela 3 apresenta os valores dos parâmetros biométricos avaliados ao fim do experimento. Houve pouca diferença entre os tratamentos sobre o desenvolvimento das mudas de Citharexylum myrianthum.

$\mathrm{Na}$ variável altura da mudas (h), o tratamento $\mathrm{T}_{2}$ e $\mathrm{T}_{1}$ foram os melhores e os tratamentos $\mathrm{T}_{5}, \mathrm{~T}_{3} \mathrm{e}$ $\mathrm{T}_{4}$ foram aqueles em que as plantas menos se desenvolveram. O parâmetro altura da parte aérea das mudas expressa a qualidade das mudas, porém deve ser analisado com outros parâmetros, como diâmetro do colo, massa seca total e relação massa seca das raízes/massa seca da parte aérea (MEXAL; LANDS, 1990; GOMES et al., 2002; CARNEIRO, 1995).

Para a variável diâmetro do colo $(\mathrm{D})$, o tratamento $\mathrm{T}_{2}$ foi superior apenas ao $\mathrm{T}_{5}$. O parâmetro diâmetro do colo é o mais observado para indicar a capacidade de sobrevivência da muda no campo e para auxiliar na definição de doses de fertilizantes a serem aplicadas na produção de mudas (CARNEIRO, 1995). Para Gomes et al. (2002), o diâmetro do colo combinado com a altura da parte aérea, por ser facilmente mensurável e não destrutivo, constitui um dos mais importantes parâmetros morfológicos para estimar o crescimento das mudas após o plantio no campo. De modo geral, a altura e o diâmetro do colo ideais das mudas para plantio no campo devem ser de $30 \mathrm{~cm}$ a $35 \mathrm{~cm}$ e $2,2 \mathrm{~cm}$ a $2,5 \mathrm{~cm}$, respectivamente. Neste caso, as mudas não apresentaram altura e diâmetro do colo ideais no momento das análises.

Já para o parâmetro comprimento de raiz (CR), não foi observado diferença estatística entre os tratamentos. Mesmo ocorrendo uma diminuição do crescimento das raízes com o aumento das proporções de pó de fumo no substrato, a DMS (5\%) é maior que essa diferença.

Em massa seca da parte aérea (MSPA) $\mathrm{T}_{2}$ e $\mathrm{T}_{1}$ foram os melhores e $\mathrm{T}_{3}, \mathrm{~T}_{4}$ e $\mathrm{T}_{5}$ não diferiram estatisticamente. Os resultados dos tratamentos $\mathrm{T}_{3}, \mathrm{~T}_{4} \mathrm{e} \mathrm{T}_{5}$ para esse parâmetro foram os mais baixos, evidenciando que as proporções de pó de fumo de 20\%, 30\% e 40\% influenciaram negativamente o desenvolvimento da planta.

Os resultados obtidos de massa seca das raízes (MSR) evidenciaram que os tratamentos $T_{1}, T_{2}$ e $T_{3}$ foram superiores aos tratamentos $\mathrm{T}_{4} \mathrm{e} \mathrm{T}_{5}$. Para Gomes (2001), a massa seca das raízes tem sido um dos mais importantes e melhores parâmetros para estimar a sobrevivência e o crescimento inicial das mudas.

Tabela 3 - Resultados dos parâmetros biométricos em mudas de Citharexylum myrianthum produzidas em sacos plásticos com substratos contendo $0 \%, 10 \%, 20 \%, 30 \%$ e $40 \%$ de pó de fumo

\begin{tabular}{lcccccc}
\hline Tratamentos & \multicolumn{5}{c}{ Parâmetros biométricos } \\
\cline { 2 - 6 } & $\begin{array}{c}\mathbf{h} \\
(\mathbf{c m})\end{array}$ & $\begin{array}{c}\mathbf{C R} \\
(\mathbf{c m})\end{array}$ & $\begin{array}{c}\text { MSPA } \\
\left(\mathrm{g} \mathrm{planta}^{-1}\right)\end{array}$ & $\begin{array}{c}\text { MSR } \\
\left(\mathbf{g ~ p l a n t a ~}^{-1}\right)\end{array}$ & $\begin{array}{c}\mathbf{D} \\
(\mathbf{m m})\end{array}$ & $\begin{array}{c}\text { MST } \\
\left(\mathrm{g} \mathrm{planta}^{-1}\right)\end{array}$ \\
\hline $\mathbf{T}_{\mathbf{1}}$ & $10,58 \mathrm{ab}$ & $6,16 \mathrm{a}$ & $0,178 \mathrm{~b}$ & $0,017 \mathrm{ab}$ & $1,34 \mathrm{ab}$ & $0,195 \mathrm{a}$ \\
$\mathbf{T}_{\mathbf{2}}$ & $11,67 \mathrm{a}$ & $5,78 \mathrm{a}$ & $0,236 \mathrm{a}$ & $0,022 \mathrm{a}$ & $1,36 \mathrm{a}$ & $0,258 \mathrm{a}$ \\
$\mathbf{T}_{\mathbf{3}}$ & $10,18 \mathrm{bc}$ & $4,41 \mathrm{a}$ & $0,117 \mathrm{c}$ & $0,013 \mathrm{bc}$ & $1,29 \mathrm{ab}$ & $0,130 \mathrm{a}$ \\
$\mathbf{T}_{\mathbf{4}}$ & $10,00 \mathrm{bc}$ & $4,60 \mathrm{a}$ & $0,124 \mathrm{c}$ & $0,009 \mathrm{~cd}$ & $1,16 \mathrm{ab}$ & $0,133 \mathrm{a}$ \\
$\mathbf{T}_{\mathbf{5}}$ & $9,20 \mathrm{c}$ & $4,07 \mathrm{a}$ & $0,105 \mathrm{c}$ & $0,004 \mathrm{~d}$ & $1,14 \mathrm{~b}$ & $0,109 \mathrm{a}$ \\
\hline $\mathbf{C V} \mathbf{( \% )}$ & 8,74 & 18,18 & 36,04 & 52,35 & 8,03 & 11,13 \\
\hline $\mathbf{D M S} \mathbf{( 5 \% )}$ & 1,19 & 2,85 & 0,02 & 0,007 & 0,22 & 2,99 \\
\hline
\end{tabular}

Nota: Médias seguidas de mesma letra não diferem estatisticamente pelo teste de Tukey a $5 \%$ de probabilidade de erro.

Legenda: $\mathrm{h}=$ altura das mudas; $\mathrm{CR}=$ comprimento de raiz; MSPA = massa seca da parte aérea; $\mathrm{MSR}=$ massa seca da raiz; $\mathrm{D}=$ diâmetro do colo; MST = massa seca total. 
A produção de massa seca total (MST) não apresentou diferença estatística entre os tratamentos, não podendo neste caso ser utilizada como parâmetro de qualidade para diferenciar os tratamentos.

Segundo Alves e Passoni (1997), a proporção de um determinado composto na formulação do substrato para a produção de mudas deve ser definida em função das exigências da espécie. Vários estudos mostram que quantidades elevadas de compostos orgânicos no substrato causam efeito negativo na produção de mudas florestais (CALDEIRA et al., 1998; CALDEIRA et al., 2007; CALDEIRA et al., 2008). Mesmo assim, dada a diversidade da flora brasileira, ainda há poucos estudos sobre a produção e o desenvolvimento de espécies nativas.

Na Tabela 4 estão apresentadas as relações entre os parâmetros morfológicos analisados e o índice de qualidade de Dickson utilizados para avaliar as diferenças entre as diferentes proporções de pó de fumo no substrato para crescimento das mudas de Citharexylum myrianthum.

Com os dados da Tabela 4, nas relações h/D e MSPA/MSR não foram observadas diferenças estatísticas entre os tratamentos, e no índice h/MSPA os tratamentos $\mathrm{T}_{1}, \mathrm{~T}_{2}$ e $\mathrm{T}_{3}$ foram os melhores, confirmando que as proporções maiores de pó de fumo no substrato não são viáveis na produção de mudas de Citharexylum myrianthum, dados semelhantes aos demais parâmetros biométricos avaliados que diferiram estatisticamente.

Tabela 4 - Relações entre os parâmetros morfológicos avaliados nas mudas de Citharexylum myrianthum produzidas com diferentes proporções do pó de fumo no substrato e o índice de qualidade de Dickson

\begin{tabular}{lcccc}
\hline \multirow{2}{*}{ Tratamentos } & \multicolumn{4}{c}{ Parâmetros biométricos } \\
\cline { 2 - 4 } & h/D & MSPA/MSR & h/MSPA & IQD \\
\hline $\mathbf{T}_{\mathbf{1}}$ & $7,92 \mathrm{a}$ & $11,77 \mathrm{a}$ & $59,70 \mathrm{a}$ & $0,89 \mathrm{a}$ \\
$\mathbf{T}_{2}$ & $8,63 \mathrm{a}$ & $12,44 \mathrm{a}$ & $50,24 \mathrm{a}$ & $0,87 \mathrm{a}$ \\
$\mathbf{T}_{\mathbf{3}}$ & $7,94 \mathrm{a}$ & $9,52 \mathrm{a}$ & $87,65 \mathrm{a}$ & $0,87 \mathrm{a}$ \\
$\mathbf{T}_{\mathbf{4}}$ & $8,92 \mathrm{a}$ & $23,21 \mathrm{a}$ & $81,59 \mathrm{~b}$ & $0,63 \mathrm{ab}$ \\
$\mathbf{T}_{5}$ & $8,08 \mathrm{a}$ & $30,37 \mathrm{a}$ & $88,64 \mathrm{~b}$ & $0,41 \mathrm{~b}$ \\
\hline $\mathbf{C V}(\mathbf{\%})$ & 5,43 & 51,25 & 23,80 & 28,67 \\
\hline $\mathbf{D M S}(\mathbf{5} \%)$ & 1,43 & 24,40 & 13,27 & 0,27 \\
\hline
\end{tabular}

Nota: Médias seguidas de mesma letra não diferem estatisticamente pelo teste de Tukey a 5\% de probabilidade de erro.

Legenda: $\mathrm{h} / \mathrm{D}=$ altura da muda/diâmetro do colo; MSPA/MSR = massa seca da parte aérea/ massa seca de raiz; h/MSPA = altura da muda/ massa seca da parte aérea; IQD = índice de qualidade de Dickson.

Para Gomes (2001), as relações MSPA/MSR e h/MSPA não são comumente usadas como índice para avaliar o padrão de qualidade de mudas, mas pode ser de grande valia se utilizadas para avaliar o potencial de sobrevivência da muda no campo. Gomes (2001) utiliza, além dos parâmetros morfológicos e suas relações, o índice de qualidade de Dickson. Segundo Carneiro (1995), o resultado da divisão da altura da parte aérea pelo diâmetro do coleto é considerado um dos mais precisos, pois fornece informações de quão delgada está a muda. E o mesmo autor relata que esse índice exprime a qualidade da muda em qualquer fase do período de produção, devendo-se se situar entre os limites 5,4 a 8,1, sendo neste caso atingido nos tratamentos $\mathrm{T}_{1}, \mathrm{~T}_{3}$ e $\mathrm{T}_{5}$.

Para o índice de qualidade de Dickson, observou-se que os tratamentos $\mathrm{T}_{1}, \mathrm{~T}_{2}, \mathrm{~T}_{3}$ e $\mathrm{T}_{4}$ não diferiram entre si quanto às médias $0,89,0,87,0,87$ e 0,63, respectivamente. Somente o $\mathrm{T}_{5}$ foi inferior, com índice 0,41. 
Em todos os tratamentos realizados obteve-se valor acima do mínimo do IQD, que é de 0,20 (DICKSON et al., 1960). O índice de qualidade de Dickson é um bom indicativo de qualidade de mudas, por considerar para o seu cálculo a robustez e o equilíbrio da distribuição da fitomassa, sendo ponderados vários parâmetros importantes (FONSECA, 2000). No entanto, para a obtenção dessa informação sobre a qualidade, existe a necessidade de proceder a métodos destrutivos da muda, onde, muitas vezes, torna-se inviável pela demanda de custo e tempo.

Tendo em vista os resultados dos parâmetros biométricos e os dados de suas relações, verifica-se que os parâmetros h, MSPA, MSR e D e a relação h/MSPA foram os que melhor representaram o desenvolvimento das mudas. $\mathrm{E}$ os tratamentos $\mathrm{T}_{4}$ e $\mathrm{T}_{5}$, em que foram utilizados $30 \%$ e $40 \%$ de pó de fumo, apresentaram os piores resultados, provavelmente graças ao $\mathrm{pH}$ elevado, que deve ter interferido no substrato - visto que os substratos apresentavam elevado $\mathrm{pH}$, essa proporção deve ter acarretado deficiências nutricionais de micronutrientes, os quais são indisponibilizados a pH acima de 6,5. Além disso, esse material pode ter aumentado a microporosidade e afetado a aeração do substrato. De acordo com Gonçalves e Poggiani (1996), quando se utiliza casca de arroz carbonizada no substrato, há uma redução da capacidade de retenção de água, além de elevar o $\mathrm{pH}$ acima de 6,5, o que pode provocar deficiências de micronutrientes.

\section{Conclusão}

As diferentes proporções do pó de fumo no substrato influenciaram o crescimento das mudas de tucaneira (Citharexylum myrianthum). As proporções de 10\% e 20\% de pó de fumo no preparo do substrato não diferiram do substrato padrão e foram as melhores na maioria dos parâmetros analisados, e os tratamentos com 30\% e 40\% de pó de fumo foram os menos viáveis, prejudicando o crescimento das mudas.

\section{Agradecimentos}

Os autores agradecem a FURB pelo apoio financeiro e a Souza Cruz S.A. por fornecer o resíduo para a pesquisa.

\section{Referências}

ALVES, W. L.; PASSONI, A. A. Composto e vermicomposto de lixo urbano na produção de mudas de oiti (Licania tomentosa Benth.) para arborização. Pesquisa Agropecuária Brasileira, v. 32, n. 10, p. 1053-1058, 1997.

CALDEIRA, M. V. W. et al. Crescimento de mudas de Eucalyptus saligna Smith em função de diferentes doses de vermicomposto. Floresta, v. 28, n. 1/2, p. 19-30, 1998.

CALDEIRA, M. V. W. et al. Influência do resíduo da indústria do algodão na formulação de substrato para a produção de mudas de Schinus terebinthifolius Raddi, Archontophoenix alexandrae Wendl. Et Drude e Archontophoenix cunninghamiana Wendl. Et Drude. Ambiência, v. 3, p. 1-8, 2007.

CALDEIRA, M. V. W. et al. Composto orgânico na produção de mudas de aroeira-vermelha. Scientia Agraria, v. 9, n. 1, p. 27-33, 2008.

CARNEIRO, J. G. A. Produção e controle de qualidade de mudas florestais. Curitiba: UFPR/FUPEF/UENF, 1995.

DICKSON, A.; LEAF, A. L.; HOSNER, J. F. Quality appraisal of white spruce and white pine seedling stock in nurseries. Forest Chronicle, v. 36, p. 10-13, 1960.

FONSECA, E. P. Padrão de qualidade de mudas de Trema micrantha (L.) Blume., Cedrela fissilis Vell. e Aspidosperma polyneuron Müll. Arg. produzidas sob diferentes períodos de sombreamento. 2000. 113 f. Tese (Doutorado em Silvicultura) - Universidade Estadual Paulista, São Paulo, 2000. 
FRANKE, I. L.; MIRANDA, E. M.; VALENTIM, J. F. Comportamento de espécies arbóreas de uso múltiplo para Sistemas Agroflorestais no estado do Acre. In: CONGRESSO BRASILEIRO DE SISTEMAS AGROFLORESTAIS, 3., 2000, Manaus. Anais... Manaus: CBSAF, 2000. v. 1, p. 97-100.

GOMES, J. M. Parâmetros morfológicos na avaliação da qualidade de mudas de Eucalyptus grandis, produzidas em diferentes tamanhos de tubetes e de dosagens de N-P-K. 2001. 126 f. Tese (Doutorado em Ciências Florestais) - Universidade Federal de Viçosa, Viçosa, MG, 2001.

GOMES, J. M. et al. Parâmetros morfológicos na avaliação da qualidade de mudas de Eucalyptus grandis. Revista Árvore, v. 26, n. 6, p. 655-664, 2002.

GONÇALVES,J.L.M.; POGGIANI, F. Substrato para produção de mudas florestais. In: SOLO-SUELO_CONGRESSO LATINO AMERICANO DE CIÊNCIA DO SOLO, 13., 1996, Águas de Lindóia. Resumos expandidos... Águas de Lindóia: SLCS/SBSC/ESALQ/USP/CEA-ESALQ/USP/SBM, 1996.

GONÇALVES, J. L. M. et al. Produção de mudas de espécies nativas: substrato, nutrição, sombreamento e fertilização. In: GONÇALVES, J. L. M.; BENEDETTI, V. (Ed.). Nutrição e fertilização florestal. Piracicaba: ESALQ/USP, 2000. p. 309-350.

LORENZI, H. Árvores brasileiras: manual de identificação e cultivo de plantas arbóreas nativas do Brasil. 2. ed. Nova Odessa, SP: Plantarum, 1998.

MEXAL, J. L.; LANDIS, T. D. Target seedling concepts: height and diameter. In: TARGET SEEDLING SYMPOSIUM, MEETING OF THE WESTERN FOREST NURSERY ASSOCIATIONS, GENERAL TECHNICAL REPORT RM-200, 1990, Roseburg. Proceedings... Fort. Collins: United States Department of Agriculture, Forest Service, 1990. p. $17-35$.

MORAIS, S. M. J. de et al. Uso do lodo de esgoto da Corsan - Santa Maria (RS), comparado com outros substratos orgânicos. Sanare, v. 6, n. 6, p. 44-49, 1996.

PORFÍRIO DA SILVA, V.; MAZUCHOWSKI, J. Z. Sistemas silvipastoris: paradigma dos pecuaristas para agregação de renda e qualidade. Curitiba: EMATER, 1999.

TRIGUEIRO, R. M. de; GERRINI, I. A. Uso de biossólido como substrato para produção de mudas de eucalipto. Scientia Forestalis, n. 64, p. 150-162, 2003.

Recebido: 25/11/2009

Received: $11 / 25 / 2009$

Aprovado: $26 / 03 / 2010$

Approved: 03/26/2010 